\title{
Los clubes deportivos de natación de Cataluña en el actual contexto competitivo del sector de las instalaciones deportivas
}

\section{Swimming sports clubs of Catalonia in the current competitive context of the sports facilities sector}

\author{
José Antonio Santacruz Lozano*1, Ángel Luis Clemente Remón' ${ }^{1}$, \\ José Emilio Jiménez-Beatty Navarro y Víctor Jiménez Díaz-Benito \\ 1 Departamento Ciencias Biomédicas, Universidad de Alcalá (España). \\ 2 Universidad Camilo José Cela (España).
}

\begin{abstract}
Resumen: Los clubes de natación son entidades sin ánimo de lucro con fines deportivos y sociales. El objetivo general del estudio fue conocer la gestión de los clubes de natación en Cataluńa cumpliendo su función social y deportiva en el actual entorno competitivo de instalaciones deportivas. Los objetivos específicos fueron conocer sus valores en indicadores de gestión, así como saber si existen diferencias significativas entre los clubes según sus espacios y oferta deportiva. La muestra del estudio ha sido formada por 19 clubes deportivos de natación de Cataluńa. El instrumento de recogida de datos fue un cuestionario adaptado a los objetivos del estudio, administrado por correo electrónico a los gerentes de cada club. Los clubes obtienen los ingresos necesarios para lograr sus fines deportivos y sociales gracias a las cuotas de sus socios. Se encontraron diferencias significativas entre ellos según los espacios y oferta deportiva en diferentes indicadores de gestión analizados.
\end{abstract}

Palabras clave: asociación deportiva; gestión del deporte; natación.
Abstract: Swimming clubs are non-profit entities for sports and social purposes. The general objective of the study was to know the management of swimming clubs in Catalonia, fulfilling their social and sports role in the current competitive environment of sports facilities. As specific objectives were to know their values on management indicators, as well as to know if significant differences between swimming clubs are found, taking in consideration their areas and sports offer. The study sample consists of 19 swimming clubs of Catalonia. The data collection instrument was a questionnaire adapted to the objectives of the study, administered by email to the managers of each club. The clubs obtain the necessary income to achieve their sporting and social goals thanks to the fees of their members. It was found significant differences between them according to the spaces and sports offer in different management indicators analyzed.

Keywords: sports association; sport management; swimming sports.

\section{Introducción}

La práctica deportiva en España ha ido aumentando en los últimos ańos. Según la Encuesta de Hábitos Deportivos en Espańa del Ministerio Educación, Cultura y Deporte (MECD, 2015) la práctica deportiva semanal española (practicar deporte o actividad física al menos una vez por semana) ha sido en 2015 del $46.2 \%$, superando a la de 2010 que fue del $37 \%$. Si bien es cierto que hay diferencias considerables entre comunidades autónomas. Un ejemplo puede ser Cataluña, donde este porcentaje es del 50.6\% en 2015 mientras que Castilla-La Mancha tiene una práctica deportiva semanal del 38.6\%. Las características de la práctica deportiva de la población están relacionadas con las propiedades del entorno del mercado, ya que la naturaleza del entorno influye en la forma organizativa del deporte, en la oferta deportiva y en el tipo práctica deportiva (organizada o libre) que realiza la población (Heinemann, 1997).

En el contexto de la práctica deportiva en instalaciones de-

Dirección para correspondencia [Correspondence address]: José Antonio Santacruz Lozano. Universidad de Alcalá (España).

Email: jose.santacruz@uah.es portivas y siguiendo los datos que proporciona la misma entidad, el $58 \%$ de la población practicante de actividad física y deporte (un 63\% en el caso de las mujeres) usa instalaciones deportivas específicas para la práctica. Y en este sentido, el número de clubes deportivos existentes en 2018 en España es de 67.512 aumentando un $0.72 \%$ a los existentes en 2017 según el Anuario de Estadísticas Deportivas (MECD, 2019). La Ley del Deporte (1990) reconoce y facilita la actividad deportiva desarrollada a través de estructuras organizativas asociativas como son los clubes deportivos. La actual legislación considera a los clubes deportivos como asociaciones privadas con personalidad jurídica propia y sin ánimo de lucro que tienen como objeto el fomento de una o varias modalidades deportivas, la práctica de actividad física y deporte de sus asociados, así como la participación en actividades y competiciones deportivas. En este sentido, es importante destacar la función social que desde la fundación de los clubes deportivos han cumplido, convirtiendo a los clubes deportivos como lugares de encuentro social entre sus asociados (Heinemann, Puig, López y Moreno, 1997; Pujadas y Santacana, 2003).

Dentro del proceso de modernización del deporte, la 
gestión de los clubes deportivos ha sufrido un desarrollo importante para parecerse a una empresa deportiva, aun siendo organizaciones sin ánimo de lucro. En este sentido, Calonge (1999) aborda el hecho de que los clubes deportivos cumplen objetivos de diferentes perspectivas: producción de servicios deportivos dirigidos a sus asociados (objetivo social); de rendimiento deportivo (objetivo deportivo); y de equilibrio en sus recursos humanos, materiales y financieros. Para ello, asegura que es necesario una organización administrativa y económica que asegure el buen funcionamiento interno del club. Cucarella (2000) sigue esta tendencia referente a los clubes deportivos asegurando que uno de los objetivos que los clubes deportivos deben plantearse es el de garantizar una gestión económica eficaz que garantice calidad en los servicios ofrecidos a sus asociados, con un nivel deportivo y social óptimo.

Es importante entender que en los clubes deportivos conviven las actividades deportivas y los entrenamientos propios de los deportes federados con los servicios de actividad física y deporte. Asimismo, la razón social del club comprende la organización de actividades sociales y culturales ofertadas a sus socios y clientes externos. Pinsach (2000) indica que el deporte federado condiciona la gestión económica de los clubes, siendo necesario la búsqueda continua de patrocinadores que aseguren la supervivencia económica del club. Cucarella (2000) profundiza más en este aspecto y propone que uno de los procesos para lograr una gestión económica eficaz es la puesta en marcha de un plan de marketing que a su vez afiance la consecución de los ingresos previstos. La estructura organizativa de un club deportivo que plantea Cucarella presenta el área de marketing con una idéntica importancia jerárquica que otras áreas como la deportiva, el área de equipamientos y el área de administración.

Centrándonos en los clubes deportivos de natación, núcleo de esta investigación, es necesario conocer las magnitudes e importancia de este deporte en el contexto general deportivo y federado. El Anuario de Estadísticas Deportivas 2019 proporciona que en 2018 se registraron en Espańa 3.866.867 licencias federadas deportivas, de las cuales el 23\% pertenecen al sexo femenino y el $77 \%$ al masculino (MECD, 2019). El deporte de la natación (con sus modalidades de natación, waterpolo, natación sincronizada, saltos, aguas abiertas y piscina corta) registró en 2018 de 67.918 licencias (1.7\% del total de licencias deportivas), incrementando 5.9\% respecto su número de licencias en 2017. La natación ocupa la posición decimotercera referente a número de licencias en el conjunto de todos los deportes federados en España. En Cataluña, en 2018 se registraron 15.556 licencias en la Federación Catalana de Natación lo que representa casi un 23\% del total de las licencias en el deporte de la natación en Espańa. El papel de la mujer en este deporte federado es muy relevante dado que representa el $48.3 \%$ de las licencias, siendo el sexto deporte con una mayor representatividad federada de la mujer, después de los deportes de gimnasia (92\%), baile deportivo (74.6\%), voleibol (73\%), hípica (69.5\%) y patinaje (62.1\%).

En referencia al número de clubes, en 2018 España tenía registrados 67.512 clubes (MECD, 2019) de los cuáles 853 clubes son de natación, lo que representa un $1.3 \%$ de la totalidad de los clubes. En Cataluña, están registrados 127 clubes de natación (Institut d'Estadística de Catalunya, 2018), lo que representaría un $14.9 \%$ de los mismos en el total de clubes de natación en España.

Conforme se ha abordado con anterioridad, además del fin deportivo y de competición, los clubes de natación cumplen con un fin social, ya que ofrecen servicios deportivos no orientados a la competición a sus socios. Este tema es de gran relevancia porque ha supuesto un gran cambio en la gestión de los clubes. Celma (2000) explica que en los últimos 20 años ha habido una transformación del deporte en los clubes deportivos incluidos los de natación, porque el concepto de socio afiliado ha sido sustituido por el concepto de socio cliente. Este hecho es la consecuencia de que los socios clientes de los clubes deportivos demandan practicar actividad física y deportes con fines de salud, lúdicos y recreativos cuando anteriormente los clubes deportivos se centraban casi exclusivamente en deporte federado. Además de este cambio en la oferta, las ayudas de la administración a los clubes deportivos pueden resultar escasa en ocasiones, lo que implica que éstos deben buscar ingresos adicionales a las subvenciones públicas para asegurar su supervivencia. Por tanto, son los socios los que ofrecen una fuente de ingresos porque están dispuestos a pagar una cantidad dineraria por practicar actividad física que satisfaga sus objetivos y expectativas (Acosta, 2005; Gambau, 2004; Mestre, Brotóns y Álvaro, 2002).

Por consiguiente, en la actualidad los clubes de natación siguen cumpliendo con una función deportiva federada y una función social para sus socios, que son quienes pagan una cantidad por practicar actividad física y deporte en las instalaciones deportivas gestionadas por los clubes de natación, asegurando así la supervivencia económica de tales entidades. Siguiendo la contribución de Puig, Moreno y López (1996) en torno a la tipología de las asociaciones, los clubes de natación de Cataluña, en algunos casos, podrían encuadrarse dentro del grupo de clubes distintivos (con instalaciones propias, equipos federados y una alta actividad social); en clubes tradicionales (donde hay un deporte principal desde donde nace la integración social) y en otros casos dentro del grupo de uniones (cuentan con elevado número de personas asociadas y donde la adhesión al club significa simpatizar con el club creando lazos de unión mediante la orientación ideológica).

La presente investigación tiene como objetivo general estudiar la función social y deportiva de los clubes de natación frente a la oferta de otras entidades de la estructura deportiva de Cataluña. 
Como objetivos específicos se plantean:

1. Conocer la cuota media mensual y el ingreso medio mensual por socio/a y de los clubes de natación a nivel global, y según sus espacios y oferta de servicios deportivos.

2. Averiguar los ingresos anuales obtenidos en 2016 procedentes de las cuotas de los socios, ingresos atípicos (ingresos que los clubes obtienen en conceptos como fisioterapia, entrenamiento personal, cursos deportivos, venta de material deportivo, entre otros) e ingresos totales de los clubes de natación tanto a nivel global como según su tipología de espacios y oferta de servicios deportivos.

3. Averiguar el porcentaje de los ingresos totales que dedican los clubes de natación a nivel global y según sus espacios y oferta deportiva a los recursos humanos, a los suministros, a la formación y a la comunicación comercial en el año 2016.

4. Conocer el número de socios (media mensual) de los clubes de natación en el año 2016 a nivel global, y según sus espacios y oferta de servicios deportivos.

5. Saber la edad media de los clientes de los clubes de natación en 2016 a nivel global, y según sus espacios y oferta de servicios deportivos.

6. Conocer la tasa de abandono (porcentaje de socios que causan baja del club respecto al total de socios) de los clubes de natación de modo global, y según sus espacios y oferta deportiva en 2016.

7. Saber el género predominante de los abonados de los clubes de natación en el año 2016 a nivel global, y según sus espacios y oferta de servicios deportivos.

8. Conocer la superficie de los clubes de natación a nivel global, y según sus espacios oferta de servicios.

9. Observar si existen diferencias significativas entre los clubes de natación según sus espacios y oferta deportiva en cualquiera de las variables de: superficie; número de usuarios; género predominante; edad; tasa de abandono; ingresos por cuotas; ingresos atípicos; porcentaje de los ingresos totales dedicados a los costes de recursos humanos, suministros formación y comunicación comercial; ingreso medio por cliente; y cuota media mensual.

\section{Material y métodos}

La investigación fue de carácter descriptivo y transversal. Partiendo de los 30 clubes de natación adscritos a la Associació Empresarial de Clubs de Natació de Catalunya (AECNC) en el año 2016, se recibió respuesta afirmativa para participar en el estudio por parte de 22 clubes. Se descartó a 3 clubes por detectar errores en la contestación al cuestionario suministrado. La muestra final del estudio fue de 19 clubes de natación con sede en la comunidad autónoma de Cataluña, siendo todos ellos miembros de la AECNC (un 59.4\% de los clubes adscritos a la AECNC). De los 19 clubes de natación que participaron, 17 gestionan una instalación deportiva con titularidad pública en régimen de concesión administrativa, y 2 clubes de natación son propietarios y gestionan su propia instalación deportiva.

Por otro lado, para poder comparar los clubes de natación según sus espacios y oferta de actividades, se dividió la muestra de 19 clubes en dos grupos diferenciados:

- Clubes tipo A: aquellos que ofrecen servicios de actividad física y deporte en el agua, en sala fitness, salas de actividades dirigidas, y servicios de otras modalidades deportivas en los que se incluyen pistas de tenis, pádel, squash y pabellón polideportivo. Este grupo está formado por 11 clubes.

- Clubes tipo B: aquellos que ofrecen servicios de actividad física y deporte en el agua, en sala fitness y en salas de actividades dirigidas. Este grupo está formado por 8 clubes.

El instrumento de recogida de los datos fue un cuestionario previamente ya empleado en varios informes de mercado del sector de las instalaciones deportivas 2015 y 2016 (Sport Managers Servicios Integrales, 2016, 2017) y que fue adaptado para este estudio. El cuestionario fue administrado por correo electrónico a los gerentes de los clubes de natación participantes que tras cumplimentarlo lo remitían también por correo electrónico a los investigadores para la codificación y tratamiento de los datos. En algunos casos fue necesaria la asistencia telefónica para la comprobación de la información recogida en el cuestionario. Este trabajo se llevó a cabo entre los meses de mayo y septiembre (ambos inclusive) de 2017 por parte de los investigadores.

Para realizar el análisis estadístico se recurrió al programa SPSS v.22.0 (SPSS Inc., EE. UU). Se utilizaron pruebas de estadística descriptiva e inferencial. En la estadística descriptiva, se hallaron datos estadísticos de frecuencia tales como: media y desviaciones típicas. En la estadística inferencial, para comprobar la normalidad de las distribuciones, se utilizó el modelo de Kolmogorov-Smirnov para N>50 (Smirnov, 1948). En el supuesto de que los datos se ajustaran a la normal, para llevar a cabo el correspondiente contraste de hipótesis se empleó el estadístico de contraste $t$ siguiendo la técnica paramétrica $t$ de student (Student, 1908) para muestras independientes. El nivel de significación se estableció en el 5\%.

\section{Resultados}

En la tabla I se observa la media de las variables estudiadas. Fueron reseńables los resultados referentes a la cuota mensual que pagan los socios de los clubes de natación de Cataluña 
que es de $30.4 €$ y los ingresos totales por club que han alcanzado los 3.680.536 € en 2016. Referente a los costes, la partida que mayor representatividad tiene fueron los recursos humanos ya que representaron casi el $45 \%$ de los ingresos totales. Con relación a indicadores de gestión, el número de socios de los clubes tiene una media de 7.854 asociados por club; la edad media de los socios fue de 41.6 años; la tasa de abandono (porcentaje de socios que causan baja del club respecto al total de socios) conformó una media del 6.5\%; y el porcentaje de mujeres como socias de los clubes fue, de media, el $48.4 \%$. Por último, sobre la superficie que ocupan los clubes deportivos, todo parece indicar que son instalaciones deportivas de gran tamańo, dado que la media de superficie útil es de $11.672 \mathrm{~m}^{2}$. Estos resultados evidencian que los clubes de natación son entidades deportivas y sociales que aglutinan un alto número de personas asociadas que disfrutan de la oferta de servicios deportivos, lúdicos y sociales.

Tabla 1. Estadísticos descriptivos de las variables de gestión analizadas.

\begin{tabular}{|c|c|c|c|c|c|}
\hline & $\mathrm{N}$ & Mínimo & Máximo & Media & Desv. Desviación \\
\hline Cuota mensual & 19 & 11,15 & 62,53 & 30,3840 & 10,85 \\
\hline Ingreso medio por usuario & 19 & 19,10 & 92,29 & 43,2959 & 17,90 \\
\hline Ingresos anuales totales & 19 & 1332844 & 9921430 & 3680536,26 & 2246012,98 \\
\hline Comunicación comercial & 19 & $0,10 \%$ & $2,95 \%$ & $1,26 \%$ & $0,78 \%$ \\
\hline Suministros & 19 & $8,40 \%$ & $18,50 \%$ & $12,20 \%$ & $3,02 \%$ \\
\hline Formación & 19 & $0,10 \%$ & $1,00 \%$ & $0,33 \%$ & $0,30 \%$ \\
\hline Externalización & 18 & $0,00 \%$ & $46,08 \%$ & $11,25 \%$ & $10,45 \%$ \\
\hline Personal & 19 & $31,00 \%$ & $61,90 \%$ & $44,89 \%$ & $7,23 \%$ \\
\hline Ingresos atípicos & 19 & 133000 & 2512594 & 1001072,32 & 682007,95 \\
\hline Ingresos por cuota & 19 & 973741 & 8066185 & 2679463,95 & 1833777,14 \\
\hline Abandono medio (\%) & 19 & $0,4 \%$ & $26 \%$ & $6,49 \%$ & $7,06 \%$ \\
\hline Mujer (\%) & 19 & $35,5 \%$ & $55 \%$ & $48,3979 \%$ & $4,79 \%$ \\
\hline Edad media & 19 & 34 & 55 & 41,56 & 4,74 \\
\hline No de socios & 19 & 2600 & 25242 & 7854,53 & 5572,96 \\
\hline Superficie $\left(\mathrm{m}^{2}\right)$ & 19 & 2900 & 50000 & 11672,21 & 11378,12 \\
\hline
\end{tabular}

La tabla II muestra las posibles diferencias entre las variables estudiadas según el tipo de club y si éstas son significativas. Se aprecia que la superficie de los clubes tipo A es mayor que la de los clubes tipo B. Además, los ingresos totales en los clubes tipo A fueron mayores que los clubes tipo B. Estos resultados se podrían explicar porque los clubes tipo A ofrecen tienen una mayor oferta de servicios deportivos. De igual modo ocurre con la variable de número de socios, donde los clubes tipo A arrojaron un mayor número de socios que los clubes tipo B, por lo que también parece lógico que los clubes tipo A obtengan mayores ingresos por cuotas y mayores ingresos por servicios atípicos. Otra diferencia a priori para tener en cuenta es que el porcentaje de mujeres socias fue mayor en los clubes tipo B que en los clubes tipo A. Respecto al porcentaje de costes dedicados a la externalización de servicios es mayor en los clubes tipo A que en los clubes tipo $\mathrm{B}$, por lo que parece que los clubes con mayor tamaño externalizan en mayor medida algunos de sus servicios. También es reseńable que la cuota mensual que pagan los socios de los clubes tipo A sea mayor que la de los clubes tipo B, e igual ocurre con el ingreso medio por socio. Entre las semejanzas entre los clubes tipo A y B, obtuvieron valores similares en las variables referentes a los porcentajes que dedican del total de sus ingresos a los costes de explotación referentes a la comunicación comercial, los costes de suministros, la formación y en los costes de personal. Entre los indicadores de gestión, la variable de tasa de abandono también tuvo cierta similitud, ya que, es del $6.4 \%$ en los clubes tipo A y de $6.7 \%$ en los clubes tipo B.

Estos resultados parecen indicar que ambos tipos de clubes dedican porcentajes similares a las partidas de coste más importantes. Adicionalmente, los clubes con mayor tamaño (tipo A) pueden ofrecer una mayor oferta en espacios y servicios a sus socios, por lo que logran tener un mayor número de socios y una cuota mensual mayor, obteniendo mayores ingresos que los clubes de menor tamaño (tipo B).

Con el objetivo de averiguar si existían diferencias significativas en las variables analizadas según los espacios y oferta de servicios deportivos (clubes deportivos tipo A y tipo B) se procedió a realizar la prueba $t$ para la igualdad de medias. En este sentido, conforme se observa en la Tabla II, se aprecian diferencias significativas entre los clubes de tipo A y tipo 
B en diferentes variables. En primer lugar, en la variable de ingresos totales $(\mathrm{p}=.001)$ donde los clubes tipo A obtienen mayores ingresos que los de tipo B. De igual modo ocurre este hecho en la variable de ingresos atípicos ( $\mathrm{p}=.009)$. También se encontraron diferencias significativas en la variable de ingresos por cuota $(\mathrm{p}=.004)$ donde los clubes tipo A tienen mayores ingresos por las cuotas de sus socios que los clubes tipo B. Por último, en la variable de porcentaje de mujeres socias del club $(\mathrm{p}=.001)$ donde los clubes tipo B tienen un porcentaje mayor de mujeres socias que los clubes tipo A. En el resto de las variables analizadas no se aprecian diferencias significativas $(\mathrm{p}>0,05)$.

Tabla 2. Media de las variables de gestión analizadas según tipo de club. Prueba t para la igualdad de medias.

\begin{tabular}{|c|c|c|c|c|c|c|c|}
\hline & \multirow{2}{*}{$\frac{\text { Tipo de club }}{\text { A }}$} & \multicolumn{4}{|c|}{ Tipo de club } & \multicolumn{2}{|r|}{ Prueba t igualdad de medias } \\
\hline & & & & $\mathrm{B}$ & & & \\
\hline & Media & $\mathrm{N}$ & Desv. & Media & $\mathrm{N}$ & Desv. & Sig. \\
\hline Cuota mensual & 32,76 & 11 & 10,95 & 27,11 & 8 & 10,43 & 0,27 \\
\hline Ingreso medio por usuario & 47,05 & 11 & 19,07 & 38,13 & 8 & 15,88 & 0,29 \\
\hline Ingresos anuales totales & 4969559 & 11 & 2153734,9 & 1908130 & 8 & 379441,05 & 0,00 \\
\hline Comunicación comercial & $1,31 \%$ & 11 & $0,97 \%$ & $1,15 \%$ & 8 & $0,48 \%$ & 0,67 \\
\hline Suministros & $12,07 \%$ & 11 & $2,77 \%$ & $12,38 \%$ & 8 & $3,52 \%$ & 0,83 \\
\hline Formación & $0,34 \%$ & 11 & $0,36 \%$ & $0,32 \%$ & 8 & $0,23 \%$ & 0,91 \\
\hline Externalización & $15,06 \%$ & 10 & $12,09 \%$ & $6,49 \%$ & 8 & $5,54 \%$ & 0,08 \\
\hline Personal & $43,13 \%$ & 11 & $6,24 \%$ & $47,31 \%$ & 8 & $8,21 \%$ & 0,22 \\
\hline Ingresos atípicos & 1329792,5 & 11 & 702658,88 & 549082 & 8 & 295718,15 & 0,01 \\
\hline Ingresos por cuota & 3639766,5 & 11 & 1893660,2 & 1359048 & 8 & 287309,83 & 0,01 \\
\hline Abandono medio (\%) & $6,35 \%$ & 11 & $7,59 \%$ & $6,68 \%$ & 8 & $6,76 \%$ & 0,92 \\
\hline Mujer (\%) & $45,59 \%$ & 11 & $4,07 \%$ & $52,26 \%$ & 8 & $2,42 \%$ & 0,01 \\
\hline Edad media & 41,64 & 11 & 5,73 & 41,45 & 8 & 3,29 & 0,94 \\
\hline No de socios & 9943,91 & 11 & 6282,58 & 4981,63 & 8 & 2680,23 & 0,05 \\
\hline Superficie $\left(\mathrm{m}^{2}\right)$ & 14940,64 & 11 & 13910,29 & 7178,13 & 8 & 4075,42 & 0,15 \\
\hline
\end{tabular}

\section{Discusión}

Este trabajo tuvo por objetivo conocer como los clubes de natación de Cataluña cumplen con sus funciones social y deportiva en el actual entorno competitivo existente entre las diferentes organizaciones deportivas. Justamente, esta investigación es pionera en los clubes sin ánimo de lucro dada la escasez de la literatura al respecto por lo que es necesario seguir estudiando el modo de organización y gestión de los clubes deportivos, que son parte importante de la estructura deportiva de España, ya que, en 2018 estaban registrados 67.512 clubes deportivos (MECD, 2019).

Si comparamos los resultados obtenidos referentes a la cuota media mensual y al ingreso medio mensual de los clubes deportivos con los obtenidos por instalaciones deportivas de titularidad privada y pública gestionadas en régimen de concesión administrativa (Sport Managers Servicios Integrales, 2017) se observa que la cuota media mensual que pagan los abonados a este tipo de instalaciones deportivas es de 30.6 $€$, cifra muy similar a la hallada en los clubes deportivos de natación de Cataluña (30.4€). Respecto al ingreso medio mensual por cliente en las instalaciones deportivas españolas ha sido de $38.1 €$ y en los clubes de natación es de $43.3 €$. En otra investigación sobre instalaciones deportivas privadas low-cost, se obtuvo que el gasto mensual que las personas realizaban abonadas a estas instalaciones deportivas está entre $19 €$ y $30 €$ mensuales (García, Gálvez, Bernal y Vélez, 2016). Estas diferencias quizá son motivadas porque los clubes de natación tienen un marcado carácter social y de sentimiento de pertenencia en sus socios que propicia la venta de servicios adicionales a la cuota mensual de socio que no se da en las instalaciones deportivas privadas low-cost.

En referencia a los ingresos, las instalaciones deportivas españolas obtuvieron en 2016 una media de ingresos totales de 1.203.913 €, cantidad que ha sido triplicada en los clubes de natación de Cataluña. Esta importante diferencia podría tener su causa en el hecho de que en el mencionado Informe de Mercado de las Instalaciones Deportivas Españolas (Sport Managers Servicios Integrales, 2017) participan todo tipo de instalación deportiva (concesiones administrativas, gimnasio de barrio, club fitness low-cost, centros de proximidad, centros deportivos polivalentes, etc.) excepto clubes deportivos 
sin ánimo de lucro e instalaciones deportivas públicas de gestión directa. De igual modo ocurre con las variables de ingresos por cuotas e ingresos atípicos, donde los clubes deportivos de natación de Cataluña presentan cantidades muy superiores a las obtenidas por las instalaciones deportivas españolas. En otra investigación de Martín (2017) sobre la situación de los clubes deportivos ilicitanos, los ingresos de los clubes deportivos, catalogados en esta investigación como clubes distintivos siguiendo la propuesta de Puig et al. (1996), obtuvieron en el año 2015 una media de $333.251 €$, cifra muy por debajo de la obtenida por los clubes de natación de Cataluña en 2016, lo que podría indicar el fuerte arraigo social y sentimiento de pertenencia que los clubes de natación catalanes tienen en sus socios.

Respecto a los costes de explotación, los recursos humanos suponen el $39.2 \%$ de los ingresos totales en las instalaciones deportivas espańolas y un $44.9 \%$ en los clubes de natación, mientras que en los clubes deportivos ilicitanos tipo selectivo (Martín, 2017) los costes de personal representan el 14.2\% de los ingresos totales. Quizá, la causa sea que el número de trabajadores en los clubes de natación de Cataluña suele ser mayor que en las instalaciones deportivas españolas y en mayor medida que en los clubes deportivos ilicitanos. El coste de los suministros parece ser más alto en los clubes de natación que en las instalaciones deportivas españolas, hecho que se pudiese explicar porque todos los clubes de natación tienen vasos de piscina. El porcentaje de los ingresos dedicados a la comunicación comercial es mayor en las instalaciones deportivas españolas que en los clubes de natación, lo que podría deberse a la necesidad mayor de comunicación comercial en las empresas privadas por el entorno tan competitivo como el que se vive actualmente en el sector de las instalaciones deportivas como ya apuntaban Boned, Felipe, Barranco, Grimaldi-Puyana y Crovetto (2015). La formación representa el $1.3 \%$ de los ingresos totales en las instalaciones deportivas españolas y un $0.3 \%$ en los clubes de natación de Cataluña, hecho que se pudiese interpretar porque las instalaciones deportivas privadas podrían tener una necesidad mayor de formación continua, pero debe ser motivo de futuras investigaciones.

En referencia a indicadores de gestión sobre características de los socios, el número de socios de los clubes es de 7.854 por club y en las instalaciones deportivas españolas es de 3.182 clientes. Esta diferencia puede deberse a que los clubes deportivos han logrado un alto sentimiento de pertenencia en su área de influencia con un arraigo social importante. Otro factor que puede influir en estas diferencias es que en el informe de las instalaciones deportivas españolas hay instalaciones deportivas de superficie mucho menor que tienen una cantidad de socios o clientes por debajo de los 1.000 socios. La tasa de abandono (porcentaje de socios que causan baja del club respecto al total de socios) tuvieron valores similares tanto los clubes de natación como las instalaciones deportivas españolas en el informe mencionado.

Con relación a las variables de edad y género, la edad media de los socios encontrada en los clubes de natación de Cataluña es algo superior la de las instalaciones deportivas españolas, 41 y 37 ańos respectivamente, y con respecto a la presencia de la mujer como socia de los clubes, parece algo mayor la presencia de la mujer en las instalaciones deportivas españolas que en los clubes de natación. Molina, Mundina y Gómez (2018) en el análisis sobre el perfil del usuario de centros deportivos privados han encontrado que no había diferencias en la variable de la edad, pero que parecía que las mujeres preferían en mayor medida que los hombres practicar actividad física o deporte en centros deportivos privados. En otra investigación de Nuviala et al. (2014) encontraron que en las instalaciones deportivas coexistían 2 grupos de clientes de similar tamańo: un primer grupo representado en mayor medida por hombre con edades entre los 16 y los 45 ańos con motivaciones hacia la práctica deportiva de deportes federados, de competición o de práctica deportiva autónoma, y un segundo grupo con mayor presencia de la mujer entre los 26 y los 65 años con motivaciones hacia la práctica deportiva en actividades lúdicas o para el aumento y mejora de la salud y de la calidad de vida. Estas diferencias podrían deberse a que las instalaciones deportivas privadas en su oferta deportiva podrían tener más en cuenta los objetivos y necesidades del público femenino que en los clubes deportivos de natación, donde la oferta de los servicios deportivos se ve influenciada por los intereses deportivos y de rendimiento del club. Otro motivo que señalaron Martín, Barriopedro, Martínez del Castillo, Jiménez-Beatty y Rivero (2014) fue que las mujeres preferían en mayor medida que los hombres las instalaciones deportivas privadas porque les ofrecían servicios para poder practicar actividad física o deporte con sus hijos o que los niños y niñas de entre 3 y 12 años tenían la opción de disfrutar de servicios lúdico-deportivos en la propia instalación deportiva de manera simultánea a la de las personas adultas. En otra investigación de Pérez-Villalba, García-Fernández, Gómez-Chacón y Fernández-Martínez (2018) encontraron que entre las personas que asistían de manera habitual a los servicios de actividades dirigidas eran en mayor medida las mujeres que los hombres. Estos resultados sugieren que en los clubes de natación analizados la oferta de actividades dirigidas no sea la óptima desde un punto de vista organizativo (horarios, tipología de actividad y espacio) puesto que en buena parte de las franjas del horario de apertura, los clubes de natación ofrecen también sus espacios de actividad al deporte federado y sus entrenamientos, no pudiendo así versatilizar su oferta de servicios conforme a los hábitos y demandas de actividad física y deporte de la población.

Por último, la superficie media de metros cuadrados útiles de los clubes de natación de Cataluña es de $11.672 \mathrm{~m}^{2}$ y en las 
instalaciones deportivas españolas es de promedio de 3.844 $\mathrm{m}^{2}$ (Sport Managers Servicios Integrales, 2017). Esta gran diferencia puede tener su explicación en que en los clubes de natación de Cataluña existen, en numerosos casos, espacios de actividad, espacios auxiliares (como por ejemplo varios núcleos de vestuarios) y singulares (como por ejemplo zonas de juego infantil o salas de lectura y televisión), que complementan y ofrecen otras posibilidades para el disfrute social $\mathrm{u}$ otras actividades relacionadas con el deporte, todas ellas diferentes a los espacios deportivos convencionales asociados a los vasos de piscina, y de fitness, como la sala fitness y salas de actividades dirigidas.

\section{Conclusiones}

En vista de estos resultados, los clubes de natación de Cataluña poseen una infraestructura orientada a cumplir sus fines deportivos y sociales. Para ello, generan una gran parte de sus ingresos procedentes de las cuotas de sus socios, que a su vez disfrutan de la práctica de actividad física y deporte con objetivos que no tienen que ver con la competición federada. Este hecho es el que asegura la supervivencia económica de los clubes de natación de Cataluña, tras la disminución en los últimos años de las subvenciones de la administración pública y los ingresos por patrocinios. Es por ello por lo que es posible concluir afirmando que los socios/as que abonan una cuota periódica, son quienes permiten a los clubes de natación continuar cumpliendo con su función deportiva (permitiendo a sus equipos y categorías federadas participar en sus competiciones y ofreciéndoles, a su vez, los espacios necesarios para sus entrenamientos) y su función social ofreciendo servicios de actividad física y deporte no federada, servicios culturales y de ocio, cuya orientación en la oferta de servicios hacen del club deportivo de natación un centro de encuentro social para sus asociados.

Asimismo, el número de socios de cada club de natación de Cataluña es alto si se compara con los clientes externos que pueden tener instalaciones deportivas públicas de gestión directa, instalaciones deportivas gestionadas en régimen de concesión administrativa o instalaciones deportivas de titularidad privada, lo cual pone de manifiesto que los clubes de natación tienen un alto grado de impacto en la sociedad, máxime en un sector tan competitivo como es el de las instalaciones deportivas en la actualidad, a la vista del crecimiento y disparidad de la oferta físico-deportiva pública y privada.

A su vez, es importante señalar que los clubes de natación de Cataluña presentan entre ellos diferencias significativas según los espacios y oferta de servicios deportivos en las variables de organización deportiva de ingresos totales, ingresos por cuotas, ingresos atípicos y porcentaje de mujeres que son socias en los clubes. Parece lógico interpretar que cuanto más espacios deportivos y mayor oferta deportiva, mayores son los ingresos (totales, por cuotas y atípicos). En este sentido, parece esencial en el proceso de planificación de explotación de los servicios deportivos de los clubes, el análisis previo de los espacios y oferta de servicios deportivos. Para explicar la diferencia en el caso de la variable del porcentaje de mujeres socias, parece oportuno seguir realizando nuevas investigaciones que amplíen la información. Sin embargo, a la vista de nuestros resultados, parece verosímil que son los clubes con espacios y oferta deportiva orientados a servicios de agua y fitness (con salas para actividades dirigidas y sala fitness) los que satisfacen mejor los objetivos y expectativas del público femenino.

El estudio presenta resultados relevantes sobre la organización y gestión de clubes deportivos de natación sin ánimo de lucro que gestionan instalaciones deportivas, pero son necesarios seguir realizando investigaciones para mejorar el conocimiento sobre la organización de este tipo de entidades deportivas. A nivel social, los resultados del estudio ponen de manifiesto la importancia social que los clubes deportivos de natación de Cataluña desarrollan siendo organizaciones que no solo ofrecen la práctica deportiva federada, sino que son entidades donde las personas asociadas acuden para cumplir con sus objetivos lúdicos, sociales, deportivos y/o de salud.

\section{Agradecimientos}

A la Associació Empresarial de Clubs de Natació de Catalunya facilitar la comunicación con los clubes de natación de Catalunya y a todos los clubes de natación que han participado en la investigación.

\section{Referencias}

1. Acosta, R. (2005). Gestión y administración de organizaciones deportivas. Barcelona: Paidotribo.

2. Boned, C.J., Felipe, J.L., Barranco, D., Grimaldi-Puyana, M. y Crovetto, M. (2015). Perfil profesional de los trabajadores de los centros de fitness en Espańa. Revista Internacional de Medicina y Ciencias de la Actividad Física y el Deporte, 15(58), 195-210.

3. Calonge, A.L. (1999). La organización y administración de clubes deportivos. Madrid: Civitas Ediciones.

4. Celma, J. (2007), Gestor y práctica deportiva: claves de futuro. En J.M. Velázquez (Eds.), La gestión deportiva local: problemática actual y tenden-

cias de futuro (pp. 135-149). Las Palmas de Gran Canaria: Federación Española de Municipios y Provincias FEMP.

5. Cucarella, A. (2000), La gestión de los clubes deportivos. En AGECEP (Eds.), ler Congreso de Gestión Deportiva de Cataluña (pp. 91-96). Barcelona: INDE.

6. Gambau, V. (2004). Estudio sobre la gestión de los clubes deportivos en Galicia. Investigación y Marketing (83), 71.

7. García Fernández, J., Gálvez Ruiz, P., Bernal García, A., y Vélez Colón, L. (2016). El gasto económico en centros de fitness low-cost: Diferencias según fidelidad y características del cliente. 
SPORT TK: Revista Euroamericana de Ciencias del Deporte, 5, 137144.

8. Heinemann, K. (1997). Aspectos sociológicos de las organizaciones deportivas. Apunts. Educación Física y Deportes (49), 10-19.

9. Heinemann, K., Puig, N., López, C. y Moreno, A. (1997). Clubs deportivos en Espańa y Alemania: una comparación teórica y empírica. Apunts. Educación Fisica y Deportes (49), 40-62.

10. Institut d'Estadística de Catalunya. (2018). Federacions Esportives 2018 Clubs y Llicències. Generalitat de Catalunya. Barcelona: Institut d'Estadística de Catalunya. Recuperado de https://www.idescat.cat/ pub/?id=aec\&n=808\&lang=es

11. Ley 10/1990, de 15 de octubre, del Deporte. Boletín Oficial del Estado, núm. 249, de 17 de octubre de 1990, pp. 30397-30411. Recuperada de https:/www.boe.es/boe/dias/1990/10/17/pdfs/A30397-30411.pdf

12. Martín, A. (2017). Situación actual de los clubes deportivos ilicitanos (Tesis doctoral). Universidad Miguel Hernández, Elche.

13. Martín, M., Barriopedro, M.L., Martínez del Castillo, J., JiménezBeatty, J.E., y Rivero, A. (2014). Diferencias de género en los hábitos de actividad física de la población adulta en la Comunidad de Madrid. RICYDE Revista Internacional de Ciencias del Deporte, X(38), 319-335.

14. Mestre, J.A., Brotóns, J.M., y Álvaro, M. (2002). La Gestión Deportiva: Clubes y Federaciones. Barcelona: INDE.

15. Ministerio Educación, Cultura y Deporte. (2015). Encuesta de Hábitos deportivos en España 2015. Madrid: Secretaría General Técnica. Subdirección General de Documentación y Publicaciones.

16. Ministerio Educación, Cultura y Deporte. (2018). Anuario Estadísticas Deportivas 2018. Madrid: Secretaría General Técnica. Subdirección General de Documentación y Publicaciones.

17. Ministerio Educación, Cultura y Deporte. (2019). Anuario Estadísticas Deportivas 2019. Madrid: Secretaría General Técnica. Subdirección General de Documentación y Publicaciones.
18. Molina, N., Mundina, J., y Gómez, A. (2018). Perfil del usuario de centros deportivos privados, según género, edad y nivel de antigüedad. SPORT TK: Revista Euroamericana de Ciencias del Deporte, 8(1), 23-28.

19. Nuviala, R., Teva-Guillén, M.R., Pérez-Ordás, R., Grao-Cruces, A., Tamayo-Fajardo, A., y Nuviala, A. (2014). Segmentación de usuarios de servicios deportivos. Retos. Nuevas tendencias en Educación Física, Deporte y Recreación, 25, 90-94.

20. Pérez-Villalba, M., García-Fernández, J., Gómez-Chacón, R., y Fernández-Martínez, N. (2018). Las actividades dirigidas en centros deportivos y su valor como elemento de fidelización. SPORT TK: Revista Euroamericana de Ciencias del Deporte, 7(2), 17-26.

21. Pinsach. J.M. (2000), Perspectivas de la gestión deportiva para entidades y clubes deportivos. En AGECEP (Eds.), ler Congreso de Gestión Deportiva de Cataluña (pp. 101-103). Barcelona: INDE.

22. Puig, N., Moreno, A., y López, C. (1996). Propuesta de marco teórico interpretativo sobre el asociacionismo deportivo en Espańa. Motricidad, 2, 75-92.

23. Pujadas, X. y Santacana, C. (2003). El club deportivo como marco de sociabilidad en España. Una visión histórica (1850-1975). Hispania, 63 (214), 505-522.

24. Smirnov, N.V. (1948). Table for estimating the goodness of fit of empirical distributions. Ann. Math. Stat, 19, 279-281.

25. Sport Managers Servicios Integrales. (2016). Informe de Mercado de las Instalaciones Deportivas Españolas 2015. Madrid: Management Around Sports.

26. Sport Managers Servicios Integrales. (2017). Informe de Mercado de las Instalaciones Deportivas Españolas 2016. Madrid: Management Around Sports.

27. Student (1908). The probable error of a mean. Biometrika, 1-25.

28 